\title{
Continuous Percolation with Discontinuities
}

\author{
Jan Nagler, ${ }^{1,2}$ Tyge Tiessen, ${ }^{1,2}$ and Harold W. Gutch ${ }^{1,3}$ \\ ${ }^{1}$ Max Planck Institute for Dynamics and Self-Organization (MPI DS) Göttingen, Germany \\ ${ }^{2}$ Institute for Nonlinear Dynamics, Faculty of Physics, University of Göttingen, Göttingen, Germany \\ ${ }^{3}$ Department of Mathematics, Technical University Munich, Munich, Germany \\ (Received 6 December 2011; revised manuscript received 19 April 2012; published 13 August 2012)
}

Complex networks are a highly useful tool for modeling a vast number of different real world structures. Percolation describes the transition to extensive connectedness upon the gradual addition of links. Whether single links may explosively change macroscopic connectivity in networks where, according to certain rules, links are added competitively has been debated intensely in the past three years. In a recent article [O. Riordan and L. Warnke, Explosive Percolation is Continuous, Science 333, 322 (2011).], O. Riordan and L. Warnke conclude that (i) any rule based on picking a fixed number of random vertices gives a continuous transition, and (ii) that explosive percolation is continuous. In contrast, we show that it is equally true that certain percolation processes based on picking a fixed number of random vertices are discontinuous, and we resolve this apparent paradox. We identify and analyze a process that is continuous in the sense defined by Riordan and Warnke but still exhibits infinitely many discontinuous jumps in an arbitrary vicinity of the transition point: a Devil's staircase. We demonstrate analytically that continuity at the first connectivity transition and discontinuity of the percolation process are compatible for certain competitive percolation systems.

DOI: 10.1103/PhysRevX.2.031009

Percolation, the transition to extensive connectedness of a network, governs the dynamics of many social and physical systems as well as in epidemic spreading of infectious diseases and information propagation [1-3]. Adding links is often not a purely random process but rather a competitive one $[4,5]$. In fact, with a spreading disease, travelers choose between one of several routes and destinations. As a result, the mobility patterns of those infected are often governed by competitive elements $[6,7]$. Consider epidemic spreading of an infectious disease. Assume that the dynamics is well described by the susceptible-infected model [8] where a population of $N$ individuals at any point in time is decomposed into susceptibles $S$ and infected $I$ such that $N=I+S$. Let the infected instantaneously transmit the disease to the susceptibles, $S \rightarrow S-1 ; I \rightarrow I+1$, according to a given contact-network dynamics [4]. A link represents an infectious contact and is introduced to the system whenever a susceptible is infected by an infected individual. The order parameter as a function of the number of links added is the size of the largest component (of infected) in the evolving (contact) network. Across all percolating systems, once the number of added links exceeds a certain critical value, the system undergoes a phase transition characterized by a sudden emergence of a giant component. In the case of contact-network dynamics, the emergence of a giant

Published by the American Physical Society under the terms of the Creative Commons Attribution 3.0 License. Further distribution of this work must maintain attribution to the author(s) and the published article's title, journal citation, and DOI.
Subject Areas: Complex Systems, Statistical Physics

component corresponds to an epidemic outbreak. Hence, the first emergence of a giant component has traditionally attracted the most attention in percolation theory $[1,2,9]$.

In contrast, multiple transitions in percolating systems have, however, been overlooked so far. Multiple phase transitions-which occur in competitive percolation, as we will demonstrate-are of high interest in various fields, particularly in geophysics and the physics of liquid crystals, but also generally in classical thermodynamics and solid state physics; see, e.g., [10-16] and references therein.

Infectious diseases, such as measles, sometimes exhibit multiple outbreaks [8]. Thus, it is not only the evolution of the size of the largest component of infected $I$ at the first transition $t_{1}$, where the number of infected becomes macroscopic for the first time, that decisively determines the spread but also its evolution after the first transition $I\left(t>t_{1}\right)$ - and all other possible successive outbreaks. The dynamics of networked systems where macroscopic components emerge abruptly is virtually impossible to predict or control, whereas systems exhibiting continuous transitions are, in principle, controllable. Hence, the type of transition is crucial for the prediction and control of many social and technological networked systems [3,17], for example, in epidemic spreading.

In a seminal paper, Achlioptas et al. [18] found strong numerical evidence for abrupt transitions in processes where links compete for addition rather than being introduced to the network purely at random. The authors called these abrupt transitions "explosive" since there was no analytical evidence that the transitions they had found numerically were truly discontinuous. Seemingly abrupt transitions involving power law divergences with very 
small exponents have been characterized as "weakly discontinuous" [5]. In contrast, truly abrupt transitions are sometimes called "most explosive" [19].

Explosive percolation has been extensively studied over the past three years in a number of follow-up works (see [5,19-30] and references therein). Most publications present further numerical evidence that such competitive processes exhibit discontinuous transitions. However, a small number of publications has emerged that presents increasing evidence that they might have continuous features $[5,21,22]$.

The question is therefore the following: Given the mechanism by which links are being added to a networked system, will the transition from the unconnected phase to global connectedness be continuous or abrupt? Very recently, Riordan and Warnke have studied the type of the first transition for a large class of percolation processes [31]. The authors grouped the rules receiving the most attention into three classes, in increasing order of generality: Achlioptas processes (AP), merging $l$-vertex (ML) rules, and general $l$-vertex (GL) rules [31-33]. Importantly, Riordan and Warnke considered two different types of continuity: continuity of the first connectivity (fc) transition, and global continuity (gc), i.e., continuity throughout the process. Their primary claim, which is made in [31], is that Achlioptas processes are fc continuous, that is, at the first connectivity transition. In [32], however, they demonstrate that processes of all three classes are fc continuous, and processes of classes AP and $\mathrm{ML}$ are also gc continuous. Riordan and Warnke state that (i) any rule based on picking a fixed number of random vertices gives a continuous transition, and (ii) that explosive percolation is continuous.

In the Achlioptas processes at each time step, two (or more) randomly chosen links compete for addition, and one of them wins the competition and is added. In this article, we focus on processes of the most general class, GL where a number of vertices are repeatedly chosen at random, and subsequently, according to a certain rule, two of the chosen vertices are linked [31]. The number $l$ of vertices to be chosen in each step is considered to be constant throughout the process. However, if $l \rightarrow \infty$ as $N \rightarrow \infty$, e.g., for $l \sim N$, then the rule that joins the two smallest distinct components involves a single discontinuous transition [5,28,31]. Such discontinuous processes are, however, necessarily trivial in the sense that the jump in the order parameter occurs at the very end of the process - as in well-known discontinuous percolation on a one-dimensional lattice [5].

We now construct a percolation process that is seemingly contradictory to both (i) and (ii). As we will see later, the process exhibits a continuous transition at the first transition point together with infinitely many discontinuous jumps in an arbitrary vicinity of the transition. Hence, competitive percolation processes are not necessarily continuous.

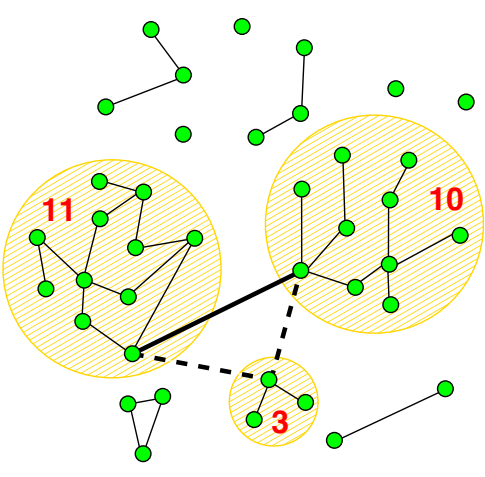

FIG. 1. Sketch of the model. Select three vertices and connect those two vertices that reside in clusters whose size difference is minimal. Here, a link between a cluster of size 10 and size 11 is established because the other two differences, $10-3=7$ and $11-3=8$, are both larger than $11-10=1$. The model does not suppress the growth of the largest cluster by definitioncontrary to many other explosive percolation processes. In contrast to what the sketch might suggest, no spatial embedding and hence no metric is involved here.

(i) Model.-Start with an empty graph $G(\mathcal{V}, \mathcal{E})$ of a finite number $N$ of vertices, $|\mathcal{V}|=N$, together with the empty set of edges $\mathcal{E}=\varnothing$. Consider the following process: At each step, three different vertices $v_{1}$, $v_{2}$, and $v_{3}$ are chosen uniformly from $\mathcal{V}$. Let $s_{1}, s_{2}$, and $s_{3}$ denote the sizes of the (not necessarily distinct) clusters in which they reside. Consider the absolute value of the differences $\Delta_{i, j}=\left|s_{i}-s_{j}\right|$, $i, j \in\{1,2,3\}$ of the cluster sizes. Connect those two vertices, $v_{i}$ and $v_{j}$, for which $\Delta_{i, j}$ is minimal (see Fig. 1). If necessary, choose randomly among multiple minima, whose corresponding vertices are to be linked. As a "final rule," when there are only two clusters left in the system, connect these. In fact, the process forbids the single largest cluster to merge with substantially smaller components. Note that the rule applies also if the vertices to be linked reside in the same component.

For a sketch of the process, see Fig. 2. Seemingly paradoxical, the case example here represents a process of the most general class, GL, which is continuous at the first transition. However, the continuity involves successive discontinuous transitions.

(ii) Resolving the paradox.-How then do we resolve the apparent paradox? We analytically demonstrate, in the following, that arbitrarily close to the onset of the continuous transition there exist infinitely many discontinuous transitions. For convenience, we make use of the Bachmann-Landau notation to characterize the size of a component $C_{i}$ in the thermodynamical limit $N \rightarrow \infty$ [9]. Components that are not macroscopic are characterized by the small- $o$ notation $C_{i}=o(N)$ such that $C_{i} / N \rightarrow 0$ as $N \rightarrow \infty$, whereas macroscopic components $C_{i}=\mathcal{O}(N)$ do not vanish 

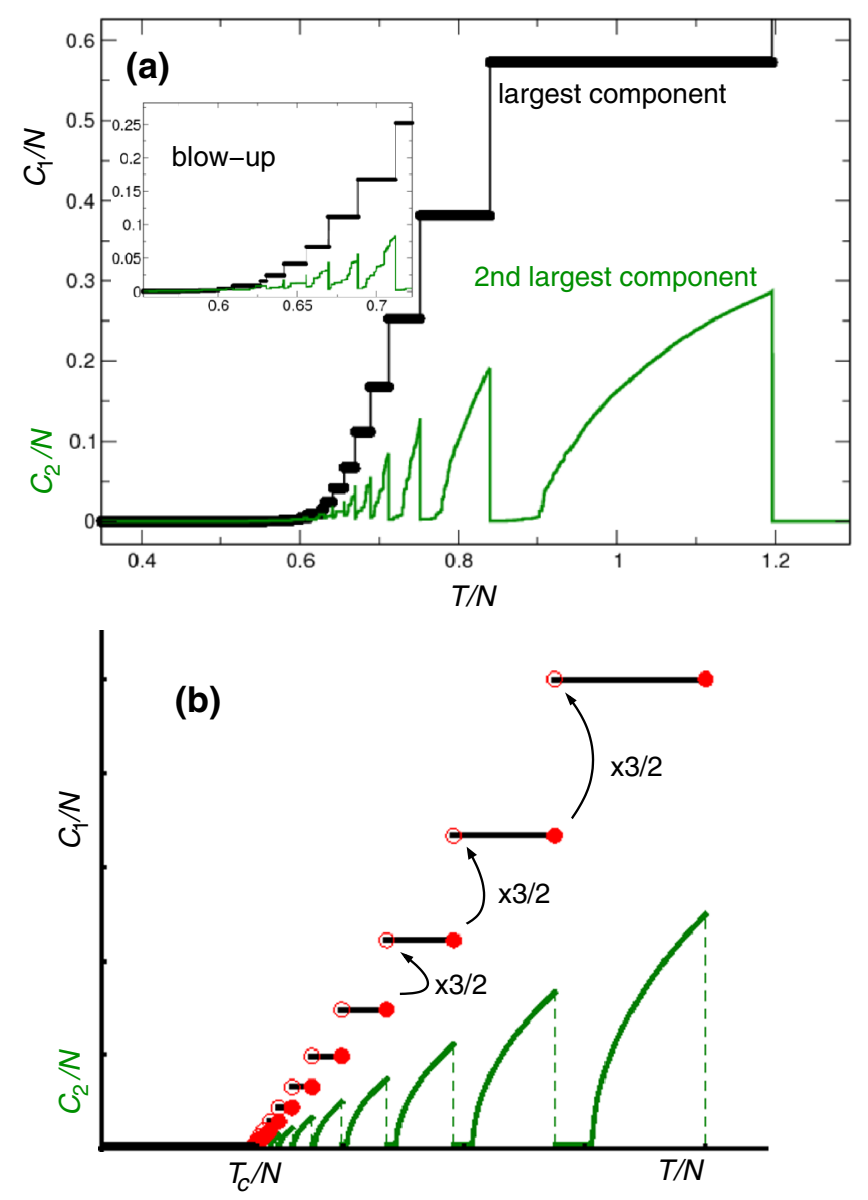

FIG. 2. The continuity of the transition and infinitely many discontinuities in any neighborhood of the onset of the phase transition are compatible. (a) Staircase shape of the multiplediscontinuous stochastic process for $N=655360$ (single realization for $l=3$ ); inset: blowup of the transition region. The evolution of the two largest components, $C_{1}$ (black curve), and $C_{2}$ (green curve), are displayed. The stairs follow approximately the rule, $C_{1} \rightarrow \frac{3}{2} C_{1}$, already established for small system sizes. The second largest component exhibits multiple continuous transitions. Mergers with the largest component involve breakdowns of $C_{2}$. In the thermodynamic limit, the process shows infinitely many discontinuous jumps but is still continuous according to the continuity property (fc) described in the text. Panel (b) shows a sketch of the staircase process for $N \rightarrow \infty$, characterizing a continuous transition at $t_{1}=T_{c} / N$ by infinitely many discontinuities.

as $C_{i} / N \rightarrow c_{0}, c_{0}>0$ as $N \rightarrow \infty$. In addition, we assume throughout the manuscript that the indices $i, j$ label the components' size rank as $C_{1} \geq C_{2} \geq$ $C_{3} \geq \ldots \geq C_{\nu}$, for some $\nu$, which is the index of the size of the smallest component.

Let us now assure ourselves of the following observation, which actually holds for any percolation process that is based on picking $l$ vertices at random from a graph. (iii) Observation 1: Upper limit for the number of macroscopic components.-With an $l$-vertex rule, there cannot exist more than $l-1$ macroscopic clusters over any extended period of time: Let $M_{\alpha}(T)$ be the number of clusters larger than $\alpha N$ at time $T$, i.e., after $T$ (time) steps, for $0<\alpha<1$. Then

$$
\frac{\left|\left\{T: M_{\alpha}(T) \geq l\right\}\right|}{N} \rightarrow 0 \quad \text { as } \quad N \rightarrow \infty,
$$

where $\left|\left\{T: M_{\alpha}(T) \geq l\right\}\right|$ denotes the number of events during the process, satisfying the condition $M_{\alpha}(T) \geq l$.

To check this observation, let us estimate the expected time that it takes until two clusters, which are at least $\alpha N$ in size, join once $M_{\alpha}(T) \geq l$. Let $\mathcal{A}$ be the event by which, in one step, two clusters, at least $\alpha N$ in size, join and let $\mathcal{B}$ be the event that we pick, in one step, all $l$ vertices from distinct clusters at least $\alpha N$ in size. When all vertices are from distinct clusters at least $\alpha N$ in size, two clusters at least $\alpha N$ in size necessarily have to merge as, in each step, two vertices have to be connected; hence, $\mathcal{B}$ implies $\mathcal{A}$. Therefore, we get

$$
\operatorname{prob}(\mathcal{A}) \geq \operatorname{prob}(\mathcal{B}) \geq \alpha^{l},
$$

where $\alpha^{l}$ is a (rough) lower bound for $\operatorname{prob}(\mathcal{B})$. This is found by picking $l$ distinct clusters at least $\alpha N$ in size, $\mathcal{C}_{1}, \mathcal{C}_{2}, \ldots, \mathcal{C}_{l}$, and then assuming the $l$ vertices are picked from these one by one.

Thus, the expected number of steps that it takes until two clusters at least $\alpha N$ in size join, given that $M_{\alpha}(T) \geq l$, is bounded from above by $1 / \alpha^{l}$. As this number of steps is independent of $N$, in rescaled time a merging of two clusters at least $\alpha N$ in size would happen instantaneously.

Furthermore, at most $1 / \alpha$ distinct clusters at least $\alpha N$ in size can be created during the process; i.e., this number is independent of $N$. Thus, the new creation of clusters at least $\alpha N$ in size cannot make up for the fast merging of these clusters.

Although, as opposed to traditional random network percolation, for $l$-vertex rules, multiple giant components can coexist [19]; the maximal number of coexisting giants, however, is limited by the parameter $l$ of the $l$-vertex rule.

In order to understand why the case example with $l=3$ exhibits the staircase shape, we now analyze how the largest component increases by the addition of single links.

(iv) Observation 2: The largest component of the system can only merge with components whose sizes are larger than or equal to half of its size (except at the end of the process).- - If this were false, we could encounter a situation where the largest cluster of size $C_{1}$ merges with a cluster that is less than $C_{1} / 2$ 
in size. But in this case the other two vertices, which are not in the largest cluster, would both have to reside in clusters less than $C_{1} / 2$ in size, and the size difference between the two smaller clusters would be less than $C_{1} / 2$ (as the size of both is already less than $C_{1} / 2$ ). Therefore, we merge the two smaller clusters (if at all), and not the largest cluster, with one of them. As a result, if the largest cluster merges at all, the size difference between any of the two smaller clusters and the largest cluster must be necessarily greater than or equal to $C_{1} / 2$.

For the following, we write with high probability (whp) to express that a certain statement does not necessarily hold for every realization of a large system, but the probability of it occurring gets arbitrarily close to 1 as $N \rightarrow \infty$. (A rigorous treatment is given in the Supplemental Material [34].)

(v) Observation 3: With high probability, the largest component cannot be overtaken when already macroscopic.-Assume that $C_{1}=\mathcal{O}(N)$. The merging of two smaller clusters, together larger than the largest component, will only take place (whp) if they are of similar size, i.e., if they exhibit the same system size scaling. This rules out (whp) overtaking [5] (at some time $T$ ) as $C_{1}(T+1)=$ $C_{i \geq 2}(T)+C_{j \geq 2}(T)$ if $C_{i \geq 2}(T)=o(N)$ but $C_{j \geq 2}(T)=$ $\mathcal{O}(N)$. Since $C_{1}$ is $\mathcal{O}(N)$, the combination $C_{i \geq 2}=o(N)$, and $C_{j \geq 2}=o(N)$, is also ruled out (whp). Therefore, a merging of two macroscopic components such that $C_{i \geq 2}=\mathcal{O}(N)$ and $C_{j \geq 2}=$ $\mathcal{O}(N)$ represents (whp) the only type of possible overtaking. This in turn requires the existence of three macroscopic components to be picked. However, from Observation 1 (cf. also [32]), we conclude that for any process based on picking $l$ vertices, the probability of the coexistence of $l$ components of size $\mathcal{O}(N)$ tends to zero as $N \rightarrow \infty$. Since we have here $l=3$, a continuous growth of $C_{1}$ is (whp) impossible when $C_{1}=\mathcal{O}(N)$.

The implication of these observations are as follows. The shape of $c_{1}(t)$ is solely given by either plateaus, or discontinuous jumps [at least $c_{1}(t) / 2$ in size], where $t=T / N$, and $c_{i}=C_{i} / N$ are scaled variables, for $N \rightarrow \infty$. The second largest component $c_{2}$-and all other smaller components $c_{i}, i>2-$ exhibit multiple continuous transitions, together with multiple breakdowns, cf. Fig. 2. In particular, mergers of $c_{1}$ and $c_{2}$ coincide with breakdowns of $c_{2}$. The evolution of the system is governed by a hierarchy of continuous transitions of smaller components: Any discontinuous transition of $c_{1}$ involves a precedent-continuous transition of $c_{2}$. Any continuous transition of $c_{2}$ involves a precedent transition of $c_{3}$, and so forth. The percolation process is discontinuous in any extended interval, which includes a small vicinity of the transition point of the first (continuous) transition.

Nevertheless, according to [31] the process must be continuous at the distinct point of the first transition $\left(t_{1}, 0\right)$ in the $\left(t, c_{1}\right)$ plane.

How many discontinuities occur?

(vi) Observation 4: The process exhibits infinitely many discontinuities in an arbitrary vicinity of the onset of the continuous transition $\left(t_{1}, 0\right)$.- - Let us parametrize the evolution of the staircase heights as $C_{1}^{(n)} \geq \frac{3}{2} C_{1}^{(n-1)}, n$ integer (see also Fig. 2). Calculate the number of discontinuous jumps $n^{\star}$ from the emergence of the largest component to the fixed macroscopic size $C_{1} / N=\beta$ for $N \rightarrow \infty$. We estimate this by the number of steps $n^{\star}$ from $C_{1}^{(0)}=\alpha N^{\gamma}$ at the phase transition where we assume sublinear asymptotics as $0<\gamma<1$ [9], to $C_{1}^{\left(n^{\star}\right)}=\beta N$, for some $\beta>\alpha$. Hence, $C_{1}^{\left(n^{\star}\right)} \geq$ $\alpha\left(\frac{3}{2}\right) n^{\star} N^{\gamma}=\beta N$, for fixed $N$, and we obtain

$$
\begin{aligned}
& n^{\star} \geq \log _{3 / 2}(\beta / \alpha)+(1-\gamma) \log _{3 / 2}(N) \rightarrow \infty, \\
& \quad \text { as } \quad N \rightarrow \infty .
\end{aligned}
$$

Since the occurrence of a transition at some $t_{1} \geq 0$, where $C_{1}$ is $\mathcal{O}(N)$ for the first time, is guaranteed [31], the staircase necessarily approaches exactly the point $\left(t_{1}, 0\right)$, as $N \rightarrow \infty$. As a result, the staircase process exhibits infinitely many discontinuous jumps arbitrarily close to the onset of the first phase transition. Interestingly, this consequence can be deduced already using only ( $\mathrm{fc}$ ) continuity and observations 13 (cf. also Supplemental Material [34]).

To conclude, we have resolved the following paradox. Riordan and Warnke have shown that all three classes of percolation processes they considered exhibit a transition that is continuous (in the mathematical sense) at one point. They have further shown that $\mathrm{AP}$ and ML processes are globally continuous but did not state anything about continuity beyond that single point for the most general class, GL. As we demonstrated above, GL processes are not necessarily globally continuous but may well exhibit (possibly infinitely many) discontinuities. In contrast to the current view, continuity at the first transition does not imply the existence of a continuous divergence, such as the power law divergences (with small exponents) that have been identified and analyzed in competitive percolation [21]. All rigorously studied percolation processes, where the largest component can merge with smaller components, have been found to exhibit single continuous transitions. In contrast, we have falsified the hypothesis that "a discontinuous transition can only occur if one avoids connecting two components that are already large" [35]. The process, 
in particular, represents the first proposed example in explosive percolation where the largest component is allowed to merge with smaller components, but where multiple-discontinuous jumps still occur.

We have focused on a particular model because it serves as a counterexample for the claim that competitive percolation is always continuous. The order parameter of the model displays a Devil's staircase with an infinite hierarchy of discontinuous jumps. To our knowledge, such a phase transition has not been reported in classical statistical mechanics of critical phenomena, except for a very recent study where the authors found strong numerical evidence for multiple jumps [36].

We have established that all processes based on picking three or more vertices at random, followed by any rule that essentially forbids the largest picked component to merge with components whose sizes are not similar, exhibit discontinuous transitions. As a result, competitive percolation is not always continuous. However, the necessary conditions for observing abrupt transitions in network percolation remain to be explored.

We cordially thank M. Timme, A. Levina, D. Tsigankov, R. D'Souza, B. Kriener, D. Lamouroux, S. Eule, and O. Trümper for valuable comments.

[1] D. Stauffer and A. Aharony, Introduction to Percolation Theory (Taylor \& Francis, London, 1994).

[2] M. Sahimi, Applications of Percolation Theory (Taylor \& Francis, London, 1994).

[3] R. Parshani, S. V. Buldyrev, H. E. Stanley, and S. Havlin, Catastrophic Cascade of Failures in Interdependent Networks, Nature (London) 464, 1025 (2010).

[4] V. Belik, T. Geisel, and D. Brockmann, Natural Human Mobility Patterns and Spatial Spread of Infectious Diseases, Phys. Rev. X 1, 0110011 (2011), and references therein.

[5] J. Nagler, A. Levina, and M. Timme, Impact of Single Links in Competitive Percolation, Nature Phys. 7, 265 (2011).

[6] L. Hufnagel, D. Brockmann, and T. Geisel, Forecast and Control of Epidemics in a Globalized World, Proc. Natl. Acad. Sci. U.S.A. 101, 15124 (2004).

[7] R. M. D'Souza, Complex Networks: Structure Comes to Random Graphs, Nature Phys. 5, 627 (2009).

[8] R. M. Anderson and R. M. May, Infectious Diseases of Humans (Oxford University Press, New York, 1991).

[9] J. Spencer, The Giant Component: The Golden Anniversary, Not. Am. Math. Soc. 57, 720 (2010).

[10] T.E. Strzelecka, M. W. Davidson, and R. L. Rill, Multiple Liquid Crystal Phases of DNA at High Concentrations, Nature (London) 331, 457 (1988).

[11] S. Honda, D. A. Yuen, S. Balachander, and D. Reuter, Three-Dimensional Instabilities of Mantle Convection with Multiple Phase Transitions, Science 259, 1308 (1993).
[12] H. Özbek, S. Yildiz, and Ö. Pekcan, Photon Transmission Technique for Studying Multiple Phase Transitions in a Liquid Crystal, Phys. Rev. E 59, 6798 (1999).

[13] A.P. Ramirez, B. S. Shastry, A. Hayashi, J. J. Krajewski, D. A. Huse, and R. J. Cava, Multiple Field-Induced Phase Transitions in the Geometrically Frustrated Dipolar Magnet: $\mathrm{Gd}_{2} \mathrm{Ti}_{2} \mathrm{O}_{7}$, Phys. Rev. Lett. 89, 067202 (2002).

[14] A. Schenck, F. N. Gygax, P. Schobinger-Papamantellos, and L. D. Tung, Multiple Magnetic Phase Transitions in $\mathrm{PrCoAl}_{4}$ Observed by Muon Spin Rotation and Relaxation Measurements, Phys. Rev. B 71, 214411 (2005).

[15] G. F. Chen, W.Z. Hu, J.L. Luo, and N. L. Wang, Multiple Phase Transitions in Single-Crystalline $\mathrm{Na}_{1-\delta} \mathrm{FeAs}$, Phys. Rev. Lett. 102, 227004 (2009).

[16] J. N. Armstrong, J. D. Felske, and H. D. Chopra, Multiple Phase Transitions Found in a Magnetic Heusler Alloy and Thermodynamics of Their Magnetic Internal Energy, Phys. Rev. B 81, 174405 (2010).

[17] Y. Liu, J.-J. Slotine, and A.-L. Barabasi, Controllability of Complex Networks, Nature (London) 473, 167 (2011).

[18] D. Achlioptas, R. M. D'Souza, and J. Spencer, Explosive Percolation in Random Networks, Science 323, 1453 (2009).

[19] W. Chen, and R. M. D'Souza, Explosive Percolation with Multiple Giant Components, Phys. Rev. Lett. 106, 115701 (2011).

[20] Y. S. Cho, B. Kahng, and D. Kim, Cluster Aggregation Model for Discontinuous Percolation Transitions Phys. Rev. E 81, 030103 (2010).

[21] R.A. da Costa, S. N. Dorogovtsev, A. V. Goltsev, and J.F.F. Mendes, Explosive Percolation Transition is Actually Continuous, Phys. Rev. Lett. 105, 255701 (2010).

[22] P. Grassberger, C. Christensen, G. Bizhani, S.-W. Son, and M. Paczuski, Explosive Percolation is Continuous, but with Unusual Finite Size Behavior, Phys. Rev. Lett. 106, 225701 (2011).

[23] F. Radicchi and S. Fortunato, Explosive Percolation in Scale-Free Networks, Phys. Rev. Lett. 103, 168701 (2009).

[24] A. A. Moreira, E. A. Oliveira, S. D. S. Reis, H. J. Herrmann, and J.S. Andrade, Hamiltonian Approach for Explosive Percolation, Phys. Rev. E 81, 040101(R) (2010).

[25] S. S. Manna and A. Chatterjee, A New Route to Explosive Percolation, Physica (Amsterdam) 390A, 177 (2011).

[26] N.A.M. Araújo and H.J. Herrmann, Explosive Percolation via Control of the Largest Cluster, Phys. Rev. Lett. 105, 035701 (2010).

[27] K. J. Schrenk, N.A. M. Araújo, and H. J. Herrmann, Gaussian Model of Explosive Percolation in Three and Higher Dimensions, Phys. Rev. E 84, 041136 (2011).

[28] E. J. Friedman and A.S. Landsberg, Construction and Analysis of Random Networks with Explosive Percolation, Phys. Rev. Lett. 103, 255701 (2009).

[29] R.M. Ziff, Explosive Growth in Biased Dynamic Percolation on Two-Dimensional Regular Lattice Networks, Phys. Rev. Lett. 103, 045701 (2009).

[30] R.M. D'Souza and M. Mitzenmacher, Local Cluster Aggregation Models of Explosive Percolation, Phys. Rev. Lett. 104, 195702 (2010). 
[31] O. Riordan and L. Warnke, Explosive Percolation is Continuous, Science 333, 322 (2011).

[32] O. Riordan and L. Warnke, Achlioptas Process Phase Transitions are Continuous, arXiv:1102.5306v2 [Ann. Appl. Probab. (to be published)].

[33] O. Riordan and L. Warnke (private communication).

[34] See Supplemental Material at http://link.aps.org/ supplemental/10.1103/PhysRevX.2.031009 for a more formal analysis.
[35] K. Panagiotou, R. Spöhel, A. Steger, and H. Thomas, Explosive Percolation in Erdös-Rényi-Like Random Graph Processes, Electronic Notes in Discrete Mathematics 38, 699 (2011).

[36] K. J. Schrenk, A. Felder, S. Deflorin, N. A. M. Araújo, R. M. D'Souza, and H. J. Herrmann, Bohman-FriezeWormald Model on the Lattice, Yielding a Discontinuous Percolation Transition, Phys. Rev. E 85, 031103 (2012). 